\title{
Prevalencia ecográfica abdominal de colicistitis en el Hospital EI Buen Samaritano, región Amazonas
}

\section{Abdominal ultrasound prevalence of colicystitis in the Good Samaritan Hospital, Amazonas region}

\author{
Edin Linares Tarrillo ${ }^{1}$, Franz Tito Coronel Zubiate ${ }^{2}$
}

\section{RESUMEN}

El presente estudio tuvo como objetivo determinar la prevalencia ecográfica abdominal de colecistitis en el Hospital El Buen Samaritano de la Provincia Bagua Grande en la Región Amazonas, Perú, en el periodo diciembre 2019 a febrero 2020. La investigación fue de enfoque cuantitativo, de nivel descriptivo, de tipo observacional, retrospectivo, transversal, y de análisis estadístico univariado. La muestra estuvo constituida por todas las historias clínicas de los pacientes atendidos. Se utilizó la técnica de observación. Los resultados fueron que de 165 pacientes atendidos con ecografía abdominal de colecistitis, solamente 59 casos nuevos presentaron una tasa de prevalencia de $35.75 \%$, la colecistitis de mayor porcentaje fue del sexo femenino con un porcentaje de $67.8 \%$, en comparación con el sexo masculino donde se obtuvo el $32.2 \%$ y la ecografía abdominal de la colecistitis aguda y crónica según la edad tuvo un mayor porcentaje en las edades mayores de 31 a 59 años con $61 \%$. Se concluye que la colecistitis afecta al sexo femenino y a edades mayores.

Palabras clave: Colecistitis, ecografía abdominal, pacientes

\begin{abstract}
The objective of this study was to determine the abdominal ultrasound prevalence of holecystitis in the Good Samaritan Hospital of the Bagua Grande Province in the Amazon Region, in the period from December 2019 to February 2020. The research was quantitative approach, descriptive level, of observational, retrospective, crosssectional type, and univariate statistical analysis. The sample consisted of all the medical records of the patients seen. The observation technique was used. The results were that of 165 patients treated with abdominal ultrasound for cholecystitis, only 59 new cases had a prevalence rate of $35.75 \%$, the highest percentage of cholecystitis was female with a percentage of $67.8 \%$, compared to male where $32.2 \%$ was obtained. and abdominal ultrasound of acute and chronic cholecystitis according to age had a higher percentage in ages older than 31 to 59 years with $61 \%$. It is concluded that cholecystitis affects the female sex and at older ages.
\end{abstract}

Keywords: Cholecystitis, abdominal ultrasound, patients

\footnotetext{
${ }^{1}$ Tesista en Tecnología Médica-Radiología, Universidad Nacional Toribio Rodríguez de Mendoza de Amazonas. Correo electrónico: edinlinarest@gmail.com

${ }^{2}$ Asesor: Docente Asociado a Tiempo Completo en la Facultad de Ciencias de la Salud, Universidad Nacional Toribio Rodríguez de Mendoza de Amazonas. Correo electrónico: franz.coronel@untrm.edu.pe
} 


\section{INTRODUCCIÓN}

La ecografía abdominal en la actualidad, es un examen que evalúa las patologías de las vías biliares, determinando el $95 \%$ de padecimientos biliares pertenecen a la colelitiasis y colecistitis (Bosch D., Schmidt J \& Kendall J, 2016, pp. 66-69).

Es un medio de diagnóstico de alta sensibilidad (97\%) y especificidad del 98\%, asimismo al precio económico. En la ecografía de cálculos tiende a presentar una estructura ecogénica envuelta de líquido biliar (Dueñas Choque, G. 2014).

Entonces a través de la ayuda de la ecografía abdominal podemos decir que la colecistitis, es un sufrimiento genético y ambiental, compartiendo genes coligados a litiasis vesicular. Además de originar casos clínicos severos y dolorosos, presentando $65 \%$ y $80 \%$ de litiasis asintomático y su diagnóstico es de forma casual en sus exámenes de ecografías, en primer lugar el cólico biliar entre 7080\% (Molina F, 2016, pp. 97-99).

Por eso la colecistitis es producto de la inflamación de la vesícula biliar y como una investigación en el resto del mundo presentando una alta prevalencia. En adultos, el $90 \%$ es producto de cálculos biliares y simplemente el 2 - $15 \%$ alitiásicos, que significa una inflamación de la vesícula sin tener cálculos denominados del mismo modo acalculosa (Gonzales G, et al, 2018, pp. 53-77).

La colecistitis se divide en dos partes: aguda y crónica. La colecistitis aguda es un problema de la colelitiasis y ataca más al sexo femenino. Su cuadro clínico principal presenta síntomas como vómitos, inapetencia y con una temperatura alta (fiebre). $\mathrm{Su}$ prescripción en el diagnóstico clínico y descubrimientos radiológicos utiliza el ultrasonido especialmente. Y el proceso de tratamiento decisivo en estos casos, se realiza la colecistectomía laparoscópica y se lleva a cabo en las primeras 24 a $72 \mathrm{~h}$ para disminuir el peligro de posibles dificultades (Molina F, 2016, pp. 97-99).

En cambio la colecistitis crónica se da por reproducción de episodios agudos por crisis estomacales, dolor de cabeza, alucinación de peso, obstinación a las frituras, estreñimiento, etc y también se da mayormente en el sexo femenino con el mismo tratamiento definitivo: la colecistectomía (Schwartz, 2010, p. 1146).

$\mathrm{Su}$ prevalencia de la colecistitis en las naciones del occidente es de $10 \%$ y el $20 \%$, y se llevan a cabo en adultos mayores y el sexo femenino. (Jerusalén C, Simón M, 2018).

Mientras en el Perú, el Hospital Nacional Arzobispo Loayza (HNAL), los pacientes con colecistitis fueron para el género femenino $(66 \%)$ en los últimos 5 años. La patología biliar fue la primera causa de morbilidad en consulta externa y hospitalización en el área de Cirugía General (Elwood MD, 2008, pp. 1241-1252).

La presente investigación tuvo como objetivo principal determinar la prevalencia ecográfica abdominal de la colecistitis en el Hospital El Buen Samaritano de la Provincia Bagua Grande, región Amazonas, periodo diciembre 2019 a Febrero 2020.

\section{MATERIAL Y MÉTODO}

La investigación fue de enfoque cuantitativo, de nivel descriptivo, de tipo observacional, retrospectivo, transversal, y de análisis estadístico univariado. La muestra estuvo constituida por todas las historias clínicas de los pacientes atendidos en el Hospital El Buen Samaritano de la Provincia Bagua Grande en la Región Amazonas, periodo diciembre 2019 a febrero 2020. Se utilizó la técnica de observación y documentación. Para el análisis clínico se utilizó una ficha de recolección de datos.

\section{RESULTADOS}

\section{Figura 1}

Prevalencia ecográfica abdominal de la colecistitis.

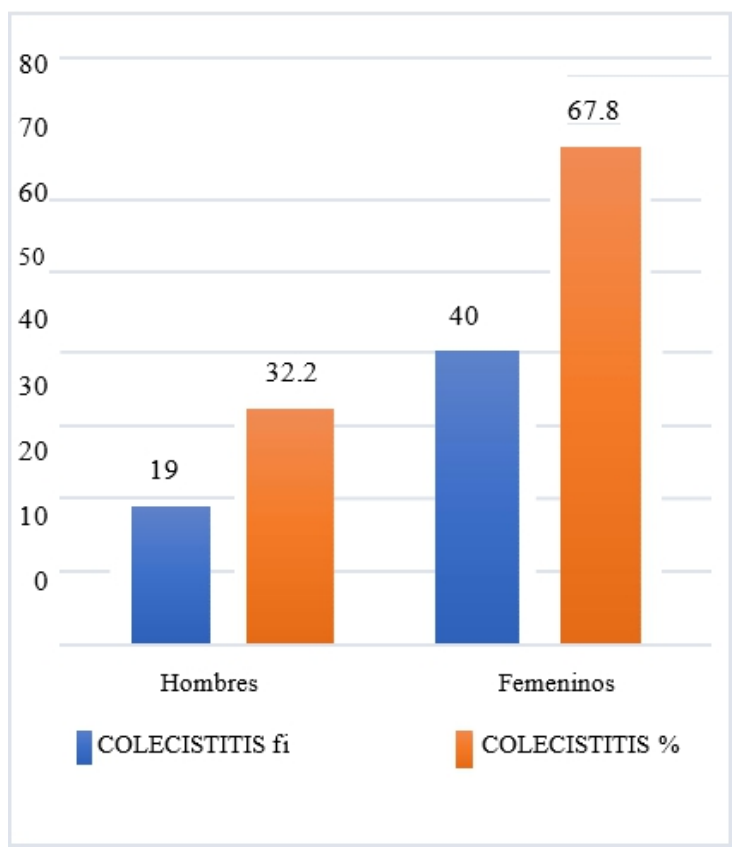

En la figura 1, muestra que la colecistitis que tuvo mayor porcentaje fue en el sexo femenino con un porcentaje de $67.8 \%$, en comparación a los hombre que presentaron un $32.2 \%$ mediante la ecografía abdominal. 


\section{Figura 2}

Prevalencia ecográfica abdominal de la colecistitis según su sexo.

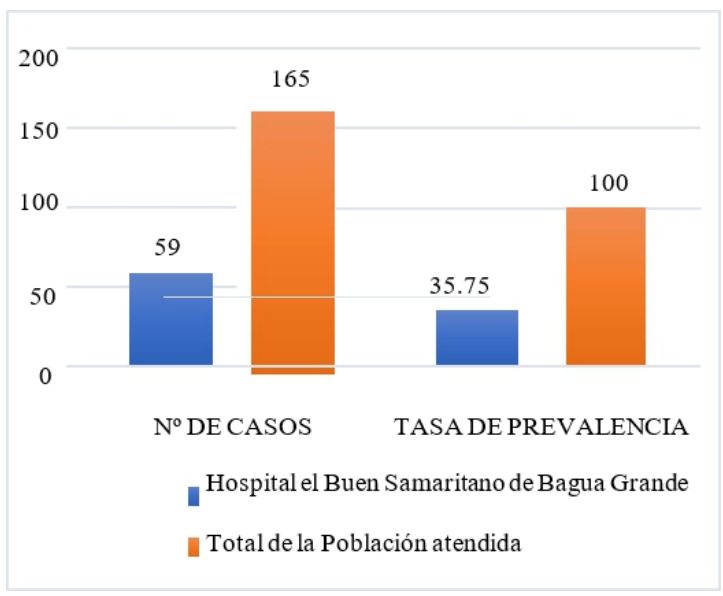

En la figura 2, muestra que de los 165 pacientes atendidos con ecografía abdominal de colecistitis en el Hospital el Buen Samaritano de Bagua Grande, solamente 59 casos nuevos presentaron una tasa de prevalencia de $35.75 \%$.

\section{Figura 3}

Prevalencia ecográfica abdominal de la colecistitis aguda y crónica según la edad.

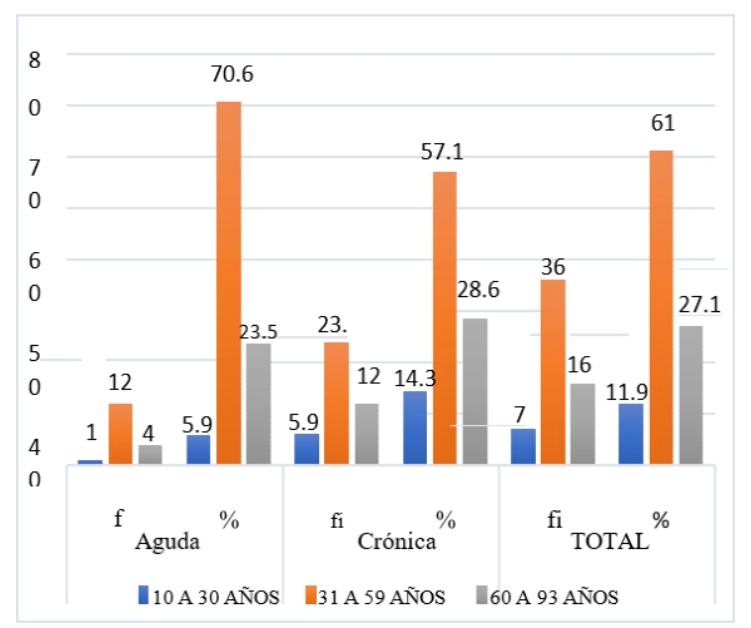

En la figura 3, presenta que la ecografía abdominal de la colecistitis aguda y crónica tuvo mayor porcentaje en las edades mayores de 31 a 59 años con $61 \%$.

\section{DISCUSIÓN}

De los hallazgos observados en la figura 1 se observa que, 165 pacientes atendidos con ecografía abdominal de colecistitis en el Hospital El Buen Samaritano, solamente 59 casos nuevos presentaron una tasa de prevalencia de $35.75 \%$.
Comparando con ciertos estudios con nuestra investigación, Cruzado (2018), realizó un estudio en el Hospital Regional en Cajamarca año 2016 y 2017, a más de 5838 pacientes atendidos en el área de Cirugía. Cuyo resultado fue que la prevalencia Ecografía Abdominal de Colecistitis en dicho Hospital, solo 406 casos de pacientes obtuvieron $38.52 \%$.

Dicho estudio mencionado, el resultado de prevalencia fue muy similar a tan solo $3 \%$ de diferencia a nuestra investigación y realizados ambos estudios en un centro hospitalario del estado, además la información requerida de ambas averiguaciones se consiguió en el área de Cirugía las historias clínicas. Resaltando que nuestro estudio a pesar del corto tiempo y con una población pequeña de habitantes, se obtuvo una tasa moderada de prevalencia de colecistitis $(35.75 \%)$, en cambio el estudio de Cruzado (2018) con una población mayor de habitantes realizados en dos años distintos con un $38.52 \%$. En un periodo de tiempo, los pacientes que serán atendidos en el Hospital El Buen Samaritano, la prevalencia comenzara a variar o tal vez presentar mayor índice de porcentaje de los casos de Colecistitis aguda o crónica.

Mientras para el estudio de Ccalachua, (2012), su muestra de estudio aleatoria fue 823 pacientes con hallazgos ecográficos Abdominal, resultando una tasa de prevalencia menor a $12.89 \%$.

Esto se debió que dichos hospitales se tomaron muestras como uno requisito de presentar un informe ecográfico en sus historias clínicas de todos los pacientes asegurados.

Pero cuando comparamos con nuestro estudio, el porcentaje de Ccalachua, (2012), fue mucho menor con un $12.89 \%$, siendo recogida la muestra de estudio en la Provincia de Arequipa todos los hospitales de Essalud, la cual podemos deducir al porcentaje menor en sus resultados. Se debió que la mayoría de los pacientes asegurados en Essalud, no contaban con sus ecografías en sus respectivas historias clínicas. En cambio, nuestro estudio, recogida las muestras de un mismo centro Hospitalario del Estado, reporto resultados en porcentajes mayores. Concluyendo que los pacientes son mejores atendidos en hospitales del estado y registrados en el sistema sus historias clínicas con sus respectivas ecografías, muy distinto para el estudio de Ccalachua.

De los hallazgos observados en la tabla y figura 02 , la Colecistitis tiene preferencia por el sexo femenino con un porcentaje de $67.8 \%$, en comparación a los hombres en menor porcentaje de $32.2 \%$ mediante la Ecografía Abdominal.

Diferido a nuestro estudio, el autor Cruzado E. (2018), repercuto igualmente una predilección para las mujeres con un $74 \%$ intervenidos en los años 2016 y 2017. Este resultado fue revalidado por 
Fernández, citado por Gonzales (2017), encontrando una relación entre femenino sobre los hombres de 4:1 con respecto a colecistitis crónica calculosa. Mientras Gonzales (2017), revela que las mujeres son más aptas a la colecistitis, resaltando que $100 \%$ de los pacientes de colecistitis, el $60 \%$ mujeres y el $40 \%$ hombres.

Y al contrastar con nuestro estudio actual, alcanzamos a llegar a una determinación que la colecistitis tiene un favoritismo significativo por las mujeres, debido que Nasser (2017), alude que tiempos atrás, la gestación y el uso extendido de los anticonceptivos orales y TRH aportan a la formación de cálculos biliares, y a su vez da como resultado a la aparición de la colecistitis.

De los hallazgos observados en la tabla y figura 03, la Ecografía Abdominal de la Colecistitis aguda y crónica tuvo mayor preferencia en las edades mayores de 31 a 59 años con $61 \%$.

Al confrontar con nuestro estudio, el autor Cruzado E. (2018), encontró edades de 15 años a 96 años, y los agrupo en tres grupos, el primero: jóvenes de 14 a 26 años, segundo: adultos de 27 a 59 años y adulto mayor de 60 a 96 años. La muestra escogida, se descubrió que la dolencia es más propensa en adultos (65\%) y ancianos con un $21.6 \%$. Este estudio fue revalidados por González (2017), afirmando en su averiguación, que la colecistitis perturba a edades de 36 a 45 años con un $50.8 \%$.

Mientras Gonzales en su tesis busca dos cosas importantes relacionar que son: causas de peligro de la colelitiasis y las tipologías litiasicas, hallando que el $56 \%$ eran mayores de 40 años. Determinando que nuestros resultados, alcanzamos a deducir que la colecistitis agudo o crónico son más vulnerables por los adultos mayores.

Y esto es avalado por Mendoza (2012). Donde afirma, que a edades mayores existe una relafile:///C:/Users/ASUS/Downloads/wwwcirugia-general- org-mx--120_Colecistitis.pdfción inmediata con la colelitiasis, al presentar un período de exhibición largo a las causas de peligro que presentan.

\section{CONCLUSIONES}

La prevalencia ecográfica abdominal de colecistitis en el Hospital El Buen Samaritano, es 35.75\%.

La prevalencia ecográfica abdominal de colecistitis es para el sexo femenino $67.8 \%$, en comparación al sexo masculino $32.2 \%$.

La mayor prevalencia ecográfica abdominal de colecistitis aguda y crónica es en las edades mayores de 31 a 59 años es $61 \%$.

\section{REFERENCIAS BIBLIOGRÁFICAS}

Bosch, D., Schmidt, JN. \& Kendall, J. (2016). Acute Cholecystitis Detected by Serial Emergency Department Focused Right Upper Quadrant Ultrasound. Journal of Medical Ultrasound; 24(2): 66 Disponible en: https://www.men del ey.com/catalogue/b298d8ab-50e1-3ebd-ad1a$54 \mathrm{~b} 8 \mathrm{e} 935 \mathrm{ba3d} /$

Bunge, M. (2014). Métodos del proceso de investigación científica. 3ra. Edic. Edit. Mexico. McGRAW-HILL / Interanericana Editores, S.A. de C.V.

Ccalachua P. (2012). Prevalencia de la patología vesicular benigna en pacientes evaluados en los hospitales de Essalud de la Provincia de Arequipa, del 1 enero 2012 hasta el 31 de diciembre del 2012. [Tesis para optar el Título Profesional de Médico Cirujano]. Universidad Nacional San Agustín, Arequipa.

Cruzado, E. (2018). Incidencia y Prevalencia de colecistitis en el servicio de Cirugía del Hospital Regional Docente de Cajamarca: Periodo 2016-2017. [Tesis para optar el Título Profesional de Médico Cirujano]. Universidad Nacional de Cajamarca, Perú.

Dueñas Choque, G. (2014). Sensibilidad y Especificidad del Estudio Ecográfico con los Hallazgos Intraoperatorios en Pacientes Colecistectomizados en el Hospital Regional Honorio Delgado Espinoza, en el periodo de Enero - Diciembre del 2013. Disponible En: Http://Tesis.Ucsm.Edu.Pe/Repositorio/Handl e/Ucsm/4843

Elwood MD. (2008). Colecistitis. Rev. Clínicas Quirúrgicas de Norteamérica; 88 (4): 12411252.Disponible en: file://C:/Users/ASUS /Downloads/www-cirugia-general- org-mx-120_Colecistitis.pdf

Gonzales RE. (2017). "Factores de riesgo asociados a pacientes con diagnóstico de colecistitis crónica calculosa en el Hospital Nacional Hipólito Unanue en el 2015'. [Tesis para optar el Título Profesional de Médico Cirujano]. Universidad Ricardo Palma, Perú.

Gonzales G, et al. (2017). Colecistitis aguda alitiasica: A propósito de un caso en paciente adolescente; 11(4): 53-57. [citado 24 de febre ro 2018.Disponible en:http://www.unsis.edu. $\mathrm{mx} / \mathrm{rev}$ ista/doc/vol4num11/6_Colecis titis.pdf 
Jerusalén C, Simón M. (2018). Cálculos biliares y sus complicaciones. Disponible en:http://ww w.aegastro.es/sites/default/files/archivos/ayud aspracticas 45 _Calculos_biliares_y _ sus _ complicaciones.pdf

Mendoza HJ. (2012). Relación entre los factores de riesgo de la colelitiasis y los tipos de litiasis en pacientes colecistectomizados Hospital Octavio Mongrut año 2010-2011. [Para obtener el Grado Académico de Magister en Docencia e Investigación en Salud]. Univers idad Nacional Mayor de San Marcos, Perú.

Molina F. (2016). Colecistitis calculosa aguda: diagnóstico y manejo. Rev. Medica de Costa Rica y Centroamericana LXXIII; 523(618): 97-99. Disponible en: https://www.medigra phic.com/pdfs/revmedcoscen/rmc-2016/r mc161s.pdf

Nasser J. (2017). Vesícula biliar y las hormonas. Disponible en: https://temassobresalud. com/vesicula-biliar-y-las hormonas/

Schwartz. (2010). Principios de cirugía. Editores, Brunicardi FC. Ed. 9a. Disponible en: https://www.academia.edu/37383336/Schwar tz_Principios_de_cirugia_9_Ed 